\title{
Symmetry Analysis and Exact Solutions to the Space-Dependent Coefficient PDEs in Finance
}

\author{
Hanze Liu ${ }^{1,2}$ \\ ${ }^{1}$ School of Mathematical Sciences, Liaocheng University, Liaocheng, Shandong 252059, China \\ ${ }^{2}$ Department of Mathematics, Binzhou University, Binzhou, Shandong 256603, China
}

Correspondence should be addressed to Hanze Liu; hzliumath@hotmail.com

Received 20 June 2013; Accepted 25 August 2013

Academic Editor: Mariano Torrisi

Copyright (C) 2013 Hanze Liu. This is an open access article distributed under the Creative Commons Attribution License, which permits unrestricted use, distribution, and reproduction in any medium, provided the original work is properly cited.

\begin{abstract}
The variable-coefficients partial differential equations (vc-PDEs) in finance are investigated by Lie symmetry analysis and the generalized power series method. All of the geometric vector fields of the equations are obtained; the symmetry reductions and exact solutions to the equations are presented, including the exponentiated solutions and the similarity solutions. Furthermore, the exact analytic solutions are provided by the transformation technique and generalized power series method, which has shown that the combination of Lie symmetry analysis and the generalized power series method is a feasible approach to dealing with exact solutions to the variable-coefficients PDEs.
\end{abstract}

\section{Introduction}

Gazizov and Ibragimov [1] studied the Black-Scholes equation of option pricing by Lie equivalence transformations. By the optimal system method, some invariant solutions to heat and Black-Scholes equations are obtained [2]. In [35], the fundamental solutions to the bond pricing equations are considered by Lie symmetry analysis and the integral transform method. In [6], the invariance properties of the bond pricing equation are studied by the group classification method. In [7], the finite element method was adopted to solve the bond pricing type of PDE system, and the numerical implementation was provided, such as system that models the TF convertible bonds with credit risk in bond pricing theory. However, the similarity reductions and exact solutions to such variable-coefficient equations are not considered generally in the aforementioned papers. Recently, we studied some nonlinear PDEs by Lie symmetry analysis and the dynamical system method [8-13]; for example, in [8], we considered Lie group classifications and exact solutions to the space-dependent coefficients hanging chain equation and the simplified bond pricing equation. In [9], we investigated the integrable condition and exact solutions to the timedependent coefficient Gardner equations by the Painlevé test and Lie group analysis method. In [10-13], we developed the generalized power series method for dealing with exact solutions to some nonlinear PDEs based on the symmetry analysis method.

It is known that the Lie symmetry analysis is a systematic and powerful method for dealing with symmetries and exact solutions to partial differential equations (see, e.g., [1-6, 818] and the references therein). Furthermore, we find that the combination of Lie symmetry analysis and the power series method is a feasible approach to investigating exact solutions to nonlinear PDEs [8-13]. On the other hand, under the perspective of mathematical physics and Lie symmetry analysis, the space-time dependent coefficients system differs greatly from its time-dependent counterpart, and it is more complicated than the latter. However, most of the studies are related to the time-dependent coefficient systems. Moreover, the determination of exact solutions to the variablecoefficients PDEs is a complicated problem that challenges researchers greatly. In the present paper, we consider the symmetry reductions and exact solutions to the general space-dependent coefficients PDEs in finance as follows:

$$
u_{t}+\alpha x^{2} u_{x x}+\beta x u_{x}+\gamma x^{v} u=0, \quad x>0,
$$

where $u=u(x, t)$ denotes the unknown function of the space variable $x$ and time $t$ and the parameters $\alpha, \beta, \gamma, \nu \in \mathbb{R}$ are arbitrary constants, $\nu \geq 0$ and $\alpha \neq 0$. 
We first note that (1) is the general form of the bond pricing types of equations [1-7]. In particular, if $v=0$, then this equation becomes the following Black-Scholes equation of option pricing:

$$
u_{t}+\alpha x^{2} u_{x x}+\beta x u_{x}+\gamma u=0, \quad x>0 .
$$

If $v=1$, then (1) is the general bond pricing equation given by

$$
u_{t}+\alpha x^{2} u_{x x}+\beta x u_{x}+\gamma x u=0, \quad x>0 .
$$

Such equations are called bond pricing types of equations, which are of great importance in financial mathematics and bond pricing theory [3-7]. For dealing with exact solutions to the variable-coefficients PDEs, we will introduce the generalized power series method [10-13] in the present paper. By a generalized power series solution, we mean a generalized power series is of the form

$$
f(\xi)=A(\xi)+\sum_{n=0}^{\infty} c_{n} \xi^{n},
$$

which is a solution to a system with respect to the variable $\xi$, where $c_{n}(n=0,1,2, \ldots)$ are constant coefficients to be determined and $A(\xi)$ is the undetermined function with respect to the variable $\xi$. In particular, if $A(\xi) \equiv 0$, then (4) is the regular power series solution. So, the generalized power series solution is the generalization of the regular power series solution and it naturally includes the latter as its special case. If we obtained a generalized power series solution (4) to a system and the convergence of this power series is shown, then the exact generalized power series solution is obtained. This solution sometimes is called the exact analytic solution $[10-13,19]$.

The main purpose of this paper is to develop the combination of Lie symmetry analysis and the generalized power series method for dealing with symmetries and exact solutions to the variable-coefficients PDEs in finance. The remainder of this paper is organized as follows. In Section 2, we perform Lie symmetry analysis on the bond pricing types of (2) and (3) and give all of the geometric vector fields of the equations in terms of the arbitrary parameters. In Section 3, we consider the symmetry reductions of the equations and provide the exponentiated solutions and the similarity solutions to the equations. In Section 4, we investigate the exact analytic solutions to the variable-coefficient equations by the generalized power series method. In Section 5, we deal with the vector fields and exact solutions to the bond pricing type of (1) for the general case $v \neq 0,1$. Finally, the conclusions and some remarks are given in Section 6.

\section{Lie Symmetry Analysis for (2) and (3)}

In this section, we will present a complete list of all possible Lie symmetry algebras for the bond pricing types of equations of the forms (2) and (3).

Recall that the geometric vector fields of such equations are as follows:

$$
V=\xi(x, t, u) \partial_{x}+\tau(x, t, u) \partial_{t}+\phi(x, t, u) \partial_{u},
$$

where $\xi(x, t, u), \tau(x, t, u)$, and $\phi(x, t, u)$ are coefficient functions of the vector field to be determined. The symmetry groups of (2) and (3) will be generated by the vector field of the form (5), respectively. Applying the second prolongation $\operatorname{pr}^{(2)} V$ of $V$ to (2) and (3), we find that the coefficient functions $\xi$, $\tau$, and $\phi$ must satisfy the following Lie symmetry condition:

$$
\left.\operatorname{pr}^{(2)} V(\Delta)\right|_{\Delta=0}=0
$$

where $\Delta=u_{t}+\alpha x^{2} u_{x x}+\beta x u_{x}+\gamma u$ for (2) and $\Delta=$ $u_{t}+\alpha x^{2} u_{x x}+\beta x u_{x}+\gamma x u$ for (3), respectively. Then, the Lie symmetry group calculation method leads to the following conditions on the coefficient functions $\xi, \tau$, and $\phi$ :

$$
\begin{aligned}
& \xi=\frac{1}{2} x \tau_{t} \log x+x \rho, \quad \phi=r(x, t) u+s(x, t), \\
& r=\frac{1}{8 \alpha} \tau_{t t} \log ^{2} x+\frac{\alpha-\beta}{4 \alpha} \tau_{t} \log x+\frac{1}{2 \alpha} \rho_{t} \log x+\sigma,
\end{aligned}
$$

for some functions $\tau, \rho$, and $\sigma$. Now the functions $\tau, \rho$, and $\sigma$ depend only on $t$. Moreover, for (2), we have

$$
\begin{aligned}
& \frac{1}{8 \alpha} \tau_{t t t} \log ^{2} x+\frac{1}{2 \alpha} \rho_{t t} \log x+\frac{1}{4} \tau_{t t} \\
& -\frac{(\alpha-\beta)^{2}-4 \alpha \gamma}{4 \alpha} \tau_{t}-\frac{\alpha-\beta}{2 \alpha} \rho_{t}+\sigma_{t}=0 ;
\end{aligned}
$$

for (3), we have

$$
\begin{aligned}
& \frac{1}{8 \alpha} \tau_{t t t} \log ^{2} x+\frac{1}{2} \gamma \tau_{t} x \log x \\
& +\frac{1}{2 \alpha} \rho_{t t} \log x+\gamma\left(\rho+\tau_{t}\right) x+\frac{1}{4} \tau_{t t} \\
& -\frac{(\alpha-\beta)^{2}}{4 \alpha} \tau_{t}-\frac{\alpha-\beta}{2 \alpha} \rho_{t}+\sigma_{t}=0 .
\end{aligned}
$$

These equations fix the functions $\xi, \tau, \rho, \sigma$, and $\phi$. Solving the equations, we obtain the vector field of (2) as follows:

$$
\begin{gathered}
V_{1}=\partial_{t}, \quad V_{2}=x \partial_{x}, \quad V_{3}=u \partial_{u}, \\
V_{4}=2 \alpha x t \partial_{x}+[\log x+(\alpha-\beta) t] u \partial_{u}, \\
V_{5}=2 \alpha x t(\log x) \partial_{x}+4 \alpha t \partial_{t} \\
+\left[(\alpha-\beta) \log x+\left((\alpha-\beta)^{2}-4 \alpha \gamma\right) t\right] u \partial_{u}, \\
V_{6}=4 \alpha x t(\log x) \partial_{x}+4 \alpha t^{2} \partial_{t} \\
+\left[2(\alpha-\beta) t \log x+\log ^{2} x-2 \alpha t\right. \\
\left.+\left((\alpha-\beta)^{2}-4 \alpha \gamma\right) t^{2}\right] u \partial_{u} \\
V_{s}=s \partial_{u},
\end{gathered}
$$

where the parameters $\alpha \neq 0, \beta, \gamma$ are arbitrary constants and the function $s=s(x, t)$ satisfies (2). 
For (3), we have the vector field as follows:

$$
V_{1}=\partial_{t}, \quad V_{2}=u \partial_{u}, \quad V_{s}=s \partial_{u},
$$

where the function $s=s(x, t)$ satisfies (3).

Clearly, for (2), a basis of the Lie algebra is $\left\{V_{1}, \ldots, V_{6}, V_{s}\right\}$. For (3), a basis for the Lie algebra is $\left\{V_{1}, V_{2}, V_{s}\right\}$. Thus, the new symmetries cannot be derived from the Lie brackets for the two equations.

Moreover, we can obtain the one-parameter groups generated by $V_{i}$, respectively. In fact, for (2), the one-parameter groups $G_{i}$ generated by $V_{i}(i=1, \ldots, 6, s)$ are given in the following:

$$
\begin{aligned}
& G_{1}:(x, t, u) \longrightarrow(x, t+\epsilon, u), \\
& G_{2}:(x, t, u) \longrightarrow\left(e^{\epsilon} x, t, u\right), \\
& G_{3}:(x, t, u) \longrightarrow\left(x, t, e^{\epsilon} u\right), \\
& G_{4}:(x, t, u) \longrightarrow\left(x e^{2 \alpha \epsilon t}, t, x^{\epsilon} u \exp \left[(\alpha-\beta) \epsilon t+\alpha \epsilon^{2} t\right]\right) \text {, } \\
& G_{5}:(x, t, u) \longrightarrow\left(x^{\delta}, \delta^{2} t, u \exp \left[\frac{\alpha-\beta}{2 \alpha}(\delta-1) \log x\right.\right. \\
& +\frac{(\alpha-\beta)^{2}-4 \alpha \gamma}{4 \alpha} \\
& \left.\left.\times\left(\delta^{2}-1\right) t\right]\right) \\
& G_{6}:(x, t, u) \\
& \longrightarrow\left(x^{1 /(1-4 \alpha \epsilon t)}, \frac{t}{1-4 \alpha \epsilon t},\right. \\
& u \sqrt{1-4 \alpha \epsilon t} \exp \left\{\left[\frac{\alpha-\beta}{2 \alpha} \log x\right.\right. \\
& +\frac{1}{4 \alpha t} \log ^{2} x \\
& \left.+\frac{(\alpha-\beta)^{2}-4 \alpha \gamma}{4 \alpha} t\right] \\
& \left.\left.\times \frac{4 \alpha \epsilon t}{1-4 \alpha \epsilon t}\right\}\right) \\
& G_{s}:(x, t, u) \longrightarrow(x, t, u+\epsilon s),
\end{aligned}
$$

where $\delta=e^{2 \alpha \epsilon}, \epsilon \ll 1$, and the function $s=s(x, t)$ is an arbitrary solution to (2). For (3), the one-parameter groups are $G_{i}(i=1,3, s)$ as above, while $s=s(x, t)$ is an arbitrary solution to (3).

From the above, we observe that $G_{1}$ is a time translation and $G_{2}$ and $G_{3}$ are trivial scaling transformations, while $G_{i}$ $(i=4,5,6)$ are nontrivial local groups of transformations. Their appearances are far from obvious from basic physical principles, but they are important for us to investigate the exact solutions to PDEs (see, e.g., $[3-5,10]$ ).

\section{Symmetry Reductions and Exact Solutions to the Bond Pricing Types of Equations}

In the preceding section, we obtained the symmetries and symmetry groups of (2) and (3). Now, we deal with the symmetry reductions and exact solutions to the equations.

3.1. The Exponentiated Solutions. Since each $G_{i}(i=$ $1, \ldots, 6, s)$ is a symmetry group, it implies that if $u=f(x, t)$ is a solution to $(2)$, then $u^{(i)}(i=1, \ldots, 6, s)$ are all solutions to the following equation as well:

$$
\begin{aligned}
& u^{(1)}=f(x, t-\epsilon) \text {, } \\
& u^{(2)}=f\left(e^{-\epsilon} x, t\right) \text {, } \\
& u^{(3)}=e^{\epsilon} f(x, t) \text {, } \\
& u^{(4)}=x^{\epsilon} \exp \left[(\alpha-\beta) \epsilon t-\alpha \epsilon^{2} t\right] f\left(e^{-2 \alpha \epsilon t} x, t\right) \text {, } \\
& u^{(5)}=\exp \left[\frac{\alpha-\beta}{2 \alpha}\left(1-\delta^{-1}\right) \log x\right. \\
& \left.+\frac{(\alpha-\beta)^{2}-4 \alpha \gamma}{4 \alpha}\left(1-\delta^{-2}\right) t\right] \\
& \times f\left(x^{1 / \delta}, \delta^{-2} t\right), \\
& u^{(6)}=\frac{1}{\sqrt{1+4 \alpha \epsilon t}} \exp \left\{\left[\frac{\alpha-\beta}{2 \alpha} \log x+\frac{1}{4 \alpha t} \log ^{2} x\right.\right. \\
& \left.\left.+\frac{(\alpha-\beta)^{2}-4 \alpha \gamma}{4 \alpha} t\right] \frac{4 \alpha \epsilon t}{1+4 \alpha \epsilon t}\right\} \\
& \times f\left(x^{1 /(1+4 \alpha \epsilon t)}, \frac{t}{1+4 \alpha \epsilon t}\right), \\
& u^{(s)}=f(x, t)+\epsilon s,
\end{aligned}
$$

where $\delta=e^{2 \alpha \epsilon}, \epsilon$ is an arbitrary real number, and the function $s=s(x, t)$ satisfies $(2)$.

For (3), the exponentiated solutions are $u^{(i)}(i=1,3, s)$ as above while $s=s(x, t)$ satisfies (3).

Such exponentiated solutions are one of group-invariant types of solutions to the PDEs, which are generated from the one-parameter groups and are of importance for studying the exact solutions and investigating the properties of solutions (see Remark 2).

Next, we investigate the symmetry reductions and exact explicit solutions to the two bond pricing equations. Firstly, we consider (2). 
3.2. Similarity Solution for $V_{1}$. For the generator $V_{1}$, we have the following reduced ordinary differential equation (ODE):

$$
\alpha \xi^{2} f^{\prime \prime}+\beta \xi f^{\prime}+\gamma f=0
$$

where $f^{\prime}=d f / d \xi$. This is an Euler equation; the corresponding characteristic equation is $\alpha K^{2}-(\alpha-\beta) K+\gamma=0$. Solving this equation, we have $K=((\alpha-\beta) \pm \sqrt{\Delta}) / 2 \alpha$, where $\Delta=(\alpha-\beta)^{2}-4 \alpha \gamma$.

When $\Delta>0$, (14) has the general solution $f=c_{1} \xi^{K_{1}}+$ $c_{2} \xi^{K_{2}}$. Thus, we obtain the exact solution to (2) as follows:

$$
u(x, t)=c_{1} x^{K_{1}}+c_{2} x^{K_{2}}
$$

where $c_{1}$ and $c_{2}$ are arbitrary constants and $K_{1,2}=((\alpha-$ $\beta) \pm \sqrt{\Delta}) / 2 \alpha$ are two real roots to the characteristic equation, respectively.

When $\Delta=0$, (14) has the general solution $f=\xi^{K}\left(c_{1}+\right.$ $\left.c_{2} \log \xi\right)$. Thus, we obtain the exact solution to (2) as follows:

$$
u(x, t)=x^{K}\left(c_{1}+c_{2} \log x\right),
$$

where $c_{1}$ and $c_{2}$ are arbitrary constants, $K=(\alpha-\beta) / 2 \alpha$ are the real root to the characteristic equation.

When $\Delta<0$, (14) has the general solution $f=$ $\xi^{K}\left(c_{1} \cos (\sqrt{-\Delta} / 2 \alpha) \log \xi+c_{2} \sin (\sqrt{-\Delta} / 2 \alpha) \log \xi\right)$. Thus, we obtain the exact solution to (2) as follows:

$$
u(x, t)=x^{K}\left(c_{1} \cos \frac{\sqrt{-\Delta}}{2 \alpha} \log x+c_{2} \sin \frac{\sqrt{-\Delta}}{2 \alpha} \log x\right),
$$

where $c_{1}$ and $c_{2}$ are arbitrary constants, $K=(\alpha-\beta) / 2 \alpha$.

3.3. Similarity Solution for $V_{2}$. For the generator $V_{2}$, we have the following reduced ODE:

$$
f^{\prime}+\gamma f=0
$$

where $f^{\prime}=d f / d \xi$. Solving this equation, we have $f=c e^{-\gamma \xi}$. Thus, we obtain the exact solution to (2) as follows:

$$
u(x, t)=c e^{-\gamma t}
$$

where $c$ is an arbitrary constant.

3.4. Similarity Solution for $V_{4}$. For the generator $V_{4}$, we have the following similarity transformation:

$$
\xi=t, \quad \omega=\log u-\frac{1}{4 \alpha t}(\log x+a t)^{2},
$$

and the similarity solution is $\omega=f(\xi)$; that is,

$$
u=\exp \left[f(t)+\frac{1}{4 \alpha t}(\log x+a t)^{2}\right] .
$$

Substituting (21) into (2), we reduce the bond pricing equation to the following ODE:

$$
2 \xi f^{\prime}+2 \gamma \xi+1=0
$$

where $f^{\prime}=d f / d \xi$. It implies that if $\omega=f(\xi)$ is a solution to (22), then (21) is a solution to (2). Solving (22), we get $f(\xi)=$ $-(1 / 2) \log \xi-\gamma \xi+c_{1}$. Thus, we obtain the solution to (2) as follows:

$$
u(x, t)=c \exp \left[\frac{1}{4 \alpha t}(\log x+a t)^{2}-\frac{1}{2} \log t-\gamma t\right],
$$

where $c$ is an arbitrary constant.

3.5. Similarity Solution for $V_{5}$. For the generator $V_{5}$, we have the following similarity transformation:

$$
\begin{gathered}
\xi=t^{-1 / 2} \log x, \\
\omega=\log u-\frac{\alpha-\beta}{2 \alpha} \log x-\frac{(\alpha-\beta)^{2}-4 \alpha \gamma}{4 \alpha} t,
\end{gathered}
$$

and the similarity solution is $\omega=f(\xi)$; that is,

$$
u=\exp \left[f\left(t^{-1 / 2} \log x\right)+\frac{\alpha-\beta}{2 \alpha} \log x+\frac{(\alpha-\beta)^{2}-4 \alpha \gamma}{4 \alpha} t\right]
$$

Substituting (25) into (2), we reduce the bond pricing equation to the following ODE:

$$
\alpha f^{\prime \prime}+\alpha f^{\prime 2}-\frac{1}{2} \xi f^{\prime}=0,
$$

where $f^{\prime}=d f / d \xi$.

Letting $f^{\prime}=y$, we get the Bernoulli equation

$$
\frac{d y}{d \xi}=\frac{1}{2 \alpha} \xi y-\alpha y^{2} \text {. }
$$

Clearly, $y=0$; that is, $f=c$ is a solution to (26). Thus, we get a solution to (2) as follows:

$$
u(x, t)=\exp \left[\frac{\alpha-\beta}{2 \alpha} \log x+\frac{(\alpha-\beta)^{2}-4 \alpha \gamma}{4 \alpha} t+c\right]
$$

for an arbitrary constant number $c$.

When $y \neq 0$, solving the Bernoulli equation, we get $y=$ $e^{(1 / 4 \alpha) \xi^{2}} /\left(\int e^{(1 / 4 \alpha) \xi^{2}} d \xi+c_{1}\right)$. Thus, we obtain the solution to (26) as follows:

$$
f(\xi)=\int \frac{e^{(1 / 4 \alpha) \xi^{2}}}{\int e^{(1 / 4 \alpha) \xi^{2}} d \xi+c_{1}} d \xi+c_{2}
$$

where $c_{1}$ and $c_{2}$ are constants of integration. Substituting (29) into (25), we obtain the exact solution to (2) immediately.

3.6. Similarity Solution for $V_{6}$. For the generator $V_{6}$, we have the following similarity transformation:

$$
\begin{aligned}
\xi= & t^{-1} \log x, \\
\omega= & \log u+\frac{1}{2} \log t-\frac{\alpha-\beta}{2 \alpha} \log x \\
& \quad-\frac{(\alpha-\beta)^{2}-4 \alpha \gamma}{4 \alpha} t-\frac{1}{4 \alpha} t^{-1} \log ^{2} x,
\end{aligned}
$$


and the similarity solution is $\omega=f(\xi)$; that is,

$$
\begin{gathered}
u=\exp \left[f\left(t^{-1} \log x\right)-\frac{1}{2} \log t+\frac{\alpha-\beta}{2 \alpha} \log x\right. \\
\left.+\frac{(\alpha-\beta)^{2}-4 \alpha \gamma}{4 \alpha} t+\frac{1}{4 \alpha} t^{-1} \log ^{2} x\right] .
\end{gathered}
$$

Substituting (31) into (2), we reduce the bond pricing equation to the following ODE:

$$
f^{\prime \prime}+f^{\prime 2}=0
$$

where $f^{\prime}=d f / d \xi$.

Solving (32), we get $f(\xi)=\log \left|\xi+c_{1}\right|+c_{3}$. Thus, we obtain the solution to (2) as follows:

$$
\begin{aligned}
u(x, t)= & c_{2}\left(\frac{1}{t} \log x+c_{1}\right) \\
& \times \exp \left[\frac{\alpha-\beta}{2 \alpha} \log x+\frac{(\alpha-\beta)^{2}-4 \alpha \gamma}{4 \alpha} t\right. \\
& \left.+\frac{1}{4 \alpha} t^{-1} \log ^{2} x-\frac{1}{2} \log t\right],
\end{aligned}
$$

where $c_{1}, c_{2}$ are arbitrary constants.

3.7. Similarity Solution for $V_{1}+v V_{2}$. For the linear combination $V=V_{1}+v V_{2}(v \neq 0$ is an arbitrary constant $)$, we have the following similarity transformation:

$$
\xi=\log x-v t, \quad \omega=u
$$

and the similarity solution is $\omega=f(\xi)$; that is,

$$
u=f(\log x-v t)
$$

Substituting (35) into (2), we reduce the bond pricing equation to the following ODE:

$$
\alpha f^{\prime \prime}-(v+\alpha-\beta) f^{\prime}+\gamma f=0,
$$

where $f^{\prime}=d f / d \xi$.

This is a second-order linear ODE; the corresponding characteristic equation is $\alpha \lambda^{2}-(v+\alpha-\beta) \lambda+\gamma=0$. Solving the algebraic equation, we have $\lambda_{1}=(v+\alpha-\beta+\sqrt{\Delta}) / 2 \alpha$, $\lambda_{2}=(v+\alpha-\beta-\sqrt{\Delta}) / 2 \alpha$, where $\Delta=(v+\alpha-\beta)^{2}-4 \alpha \gamma$.

When $\Delta>0$, (36) has the solution $f(\xi)=c_{1} e^{\lambda_{1} \xi}+c_{2} e^{\lambda_{2} \xi}$. Thus, we obtain the solution to (2) as follows:

$$
u(x, t)=c_{1} x^{\lambda_{1}} e^{-\lambda_{1} v t}+c_{2} x^{\lambda_{2}} e^{-\lambda_{2} v t}
$$

where $c_{1}, c_{2}$ are arbitrary constants.
When $\Delta=0$, (36) has the solution $f(\xi)=\left(c_{1}+c_{2} \xi\right) e^{\lambda \xi}$, where $\lambda=(v+\alpha-\beta) / 2 \alpha$. Thus, we obtain the solution to (2) as follows:

$$
u(x, t)=\left[c_{1}+c_{2}(\log x-v t)\right] x^{\lambda} e^{-\lambda v t},
$$

where $c_{1}, c_{2}$ are arbitrary constants.

When $\Delta<0$, (36) has the solution $f(\xi)=$ $\left(c_{1} \cos (\sqrt{-\Delta} / 2 \alpha) \xi+c_{2} \sin (\sqrt{-\Delta} / 2 \alpha) \xi\right) e^{((v+\alpha-\beta) / 2 \alpha) \xi}$. Thus, we obtain the solution to (2) as follows:

$$
\begin{aligned}
u(x, t)= & x^{(v+\alpha-\beta) / 2 \alpha} e^{-(v(v+\alpha-\beta) / 2 \alpha) t} \\
\times & {\left[c_{1} \cos \frac{\sqrt{-\Delta}}{2 \alpha}(\log x-v t)\right.} \\
& \left.\quad+c_{2} \sin \frac{\sqrt{-\Delta}}{2 \alpha}(\log x-v t)\right],
\end{aligned}
$$

where $c_{1}, c_{2}$ are arbitrary constants.

3.8. Similarity Reduction for $V_{1}+v V_{3}$. For the linear combination $V=V_{1}+v V_{3}(v \neq 0$ is an arbitrary constant $)$, we have the following similarity transformation:

$$
\xi=x, \quad \omega=\log u-v t,
$$

and the similarity solution is $\omega=f(\xi)$; that is,

$$
u=\exp \{f(x)+v t\} .
$$

Substituting (41) into (2), we reduce the bond pricing equation to the following ODE:

$$
\alpha \xi^{2} f^{\prime \prime}+\alpha \xi^{2} f^{\prime 2}+\beta \xi f^{\prime}+v+\gamma=0,
$$

where $f^{\prime}=d f / d \xi$. This is a nonlinear second-order ODE. In the next section, we will deal with such an equation by the special transformation technique.

3.9. Similarity Solution for $V_{2}+v V_{3}$. For the linear combination $V=V_{2}+v V_{3}(v \neq 0$ is an arbitrary constant $)$, we have the following similarity transformation:

$$
\xi=t, \quad \omega=x^{-v} u,
$$

and the similarity solution is $\omega=f(\xi)$; that is,

$$
u=x^{v} f(t) \text {. }
$$

Substituting (44) into (2), we reduce the bond pricing equation to the following ODE:

$$
f^{\prime}+\alpha v(v-1) f+\beta v f+\gamma f=0,
$$

where $f^{\prime}=d f / d \xi$.

Solving (45), we get $f=c \exp \left\{-\left[\alpha v^{2}-(\alpha-\beta) v+\gamma\right] \xi\right\}$. Thus, we obtain the solution to (2) as follows

$$
u(x, t)=c x^{v} \exp \left\{-\left[\alpha v^{2}-(\alpha-\beta) v+\gamma\right] t\right\},
$$

where $c$ is an arbitrary constant. 
3.10. Similarity Reduction for $V_{1}+v V_{4}$. For the linear combination $V=V_{1}+v V_{4}(v \neq 0$ is an arbitrary constant), we have the following similarity transformation:

$$
\begin{gathered}
\xi=\log x-v \alpha t^{2} \\
\omega=\log u-v t \log x+\frac{2}{3} \alpha v^{2} t^{3}-\frac{1}{2}(\alpha-\beta) v t^{2},
\end{gathered}
$$

and the similarity solution is $\omega=f(\xi)$; that is,

$$
\begin{gathered}
u=\exp \left[f\left(\log x-v \alpha t^{2}\right)+v t \log x-\frac{2}{3} \alpha v^{2} t^{3}\right. \\
\left.+\frac{1}{2}(\alpha-\beta) v t^{2}\right] .
\end{gathered}
$$

Substituting (48) into (2), we reduce the bond pricing equation to the following ODE:

$$
\alpha f^{\prime \prime}+\alpha f^{\prime 2}-(\alpha-\beta) f^{\prime}+v \xi+\gamma=0
$$

where $f^{\prime}=d f / d \xi$. This is a nonlinear second-order ODE also. In the next section, we will deal with the exact solutions to such equations.

Secondly, we consider (3). In fact, for this equation, we have the nontrivial cases as follows only.

3.11. Similarity Reduction for $V_{1}$ of (3). For the generator $V_{1}$, we have the following reduced ordinary differential equation (ODE):

$$
\alpha \xi f^{\prime \prime}+\beta f^{\prime}+\gamma f=0
$$

where $f^{\prime}=d f / d \xi$. This is a nonlinear second-order ODE as well; there is no general method for tackling it yet. In Section 4, we will deal with such equations by the power series method.

3.12. Similarity Reduction for $V_{1}+v V_{2}$ of (3). For the linear combination $V=V_{1}+v V_{2}(v \neq 0$ is an arbitrary constant), we have the following similarity transformation:

$$
\xi=x, \quad \omega=\log u-v t
$$

and the similarity solution is $\omega=f(\xi)$; that is,

$$
u=\exp [f(x)+v t]
$$

Substituting (52) into (3), we reduce the second bond pricing equation to the following ODE:

$$
\alpha \xi^{2} f^{\prime \prime}+\alpha \xi^{2} f^{\prime 2}+\beta \xi f^{\prime}+\gamma \xi+v=0,
$$

where $f^{\prime}=d f / d \xi$. This is a nonlinear second-order ODE also. Similar to the above equations, we will deal with such equations by the generalized power series method in the next section.

\section{Exact Analytic Solutions in terms of the Generalized Power Series Method}

In Section 3, we considered the symmetry reductions and exact solutions to the bond pricing types of (2) and (3). In this section, we will deal with the nonlinear ODEs (42), (49), (50), and (53) by the special transformation technique and generalized power series method. Thus, the exact analytic solutions to (2) and (3) are obtained.

4.1. Exact Solution to (2). Firstly, we consider the ODE (42). Letting $f^{\prime}=y$, we get the Riccati equation

$$
\frac{d y}{d \xi}=-y^{2}-\frac{\beta}{\alpha \xi} y-\frac{v+\gamma}{\alpha \xi^{2}} .
$$

Now, we solve the equation by the transformation technique directly. Suppose that (54) has the solution of the form

$$
y=p \xi^{-1}
$$

where $p$ is a constant to be determined. Substituting (55) into (54), we have $\alpha p^{2}-(\alpha-\beta) p+v+\gamma=0$. Solving the algebraic equation, we get

$$
p=\frac{(\alpha-\beta) \pm \sqrt{\Delta}}{2 \alpha}
$$

where $\Delta=(\alpha-\beta)^{2}-4 \alpha(v+\gamma)$.

Setting $y=z+p \xi^{-1}$ and plugging it into (54), we get

$$
\frac{d z}{d \xi}=-z^{2}-q \frac{z}{\xi}, \quad q=2 p+\frac{\beta}{\alpha} .
$$

This is a Bernoulli equation. Solving the equation, we have the following results.

When $q=1$, we get $f(\xi)=p \log \xi+\log \left(\log \xi+c_{1}\right)+c_{2}$. Thus, the exact solution to (2) is

$$
u(x, t)=c_{2} x^{p}\left(\log x+c_{1}\right) e^{v t}
$$

where $c_{1}$ and $c_{2}$ are arbitrary constants; $p$ and $q$ are given by (56) and (57).

When $q \neq 1$, we get $f(\xi)=p \log \xi+(1-q) \int d \xi /(\xi+$ $\left.c_{1} \xi^{q}\right)+c_{2}$. Thus, the exact solution to (2) is

$$
u(x, t)=c_{2} x^{p} \exp \left[(1-q) \int \frac{d x}{x+c_{1} x^{q}}+v t\right],
$$

where $c_{1}$ and $c_{2}$ are arbitrary constants and $p$ and $q$ are given by (56) and (57).

4.2. Exact Analytic Solution to (2). Through the transformation technique, we solve the Riccati equation (54), so the exact solutions to (2) are obtained. But for the other equations such as (49), (50), and (53), we cannot get the exact solutions by such special transformation technique. However, we know that the power series can be used to solve nonlinear ODEs, including many complicated differential equations with nonconstant coefficients [10-13, 19, 20]. 
Now, we consider the power series solution to the reduced equation (49). Letting $f^{\prime}=y$, we get the following Riccati equation:

$$
\alpha y^{\prime}+\alpha y^{2}-(\alpha-\beta) y+v \xi+\gamma=0
$$

We will seek a solution of (60) in a power series of the form

$$
y=\sum_{n=0}^{\infty} c_{n} \xi^{n}=p+\sum_{n=1}^{\infty} c_{n} \xi^{n}, \quad p=c_{0}
$$

where the coefficients $c_{n}(n=0,1,2, \ldots)$ are constants to be determined.

Substituting (61) into (60) and comparing coefficients, we obtain

$$
\begin{gathered}
c_{1}=-p^{2}+\frac{\alpha-\beta}{\alpha} p-\frac{\gamma}{\alpha}, \\
c_{2}=-p c_{1}+\frac{\alpha-\beta}{2 \alpha} c_{1}-\frac{v}{2 \alpha} .
\end{gathered}
$$

Generally, for $n \geq 2$, we have

$$
c_{n+1}=\frac{1}{(n+1) \alpha}\left[(\alpha-\beta) c_{n}-\alpha \sum_{k=0}^{n} c_{k} c_{n-k}\right] \text {. }
$$

Thus, for arbitrarily choosing the parameter $c_{0}$, from (62), we can get $c_{1}$ and $c_{2}$. Furthermore, in view of (63), we have

$$
\begin{aligned}
& c_{3}=\frac{\alpha-\beta}{3 \alpha} c_{2}-\frac{1}{3}\left(2 p c_{2}+c_{1}^{2}\right), \\
& c_{4}=\frac{\alpha-\beta}{4 \alpha} c_{3}-\frac{1}{2}\left(p c_{3}+c_{1} c_{2}\right),
\end{aligned}
$$

and so on.

Therefore, the other terms of the sequence $\left\{c_{n}\right\}_{n=0}^{\infty}$ can be determined successively from (63) in a unique manner. This implies that for (60) there exists a power series solution (61) with the coefficients given by (62) and (63). Furthermore, we can show the convergence of the power series solution (61) with the coefficients given by (62) and (63) (see, e.g., [10, 12, $13,19])$; the details are omitted here. So, this solution (61) to (60) is an exact analytic solution.

Hence, the exact power series solution to (49) can be written as follows:

$$
f(\xi)=c+p \xi+\frac{1}{2} c_{1} \xi^{2}+\frac{1}{3} c_{2} \xi^{3}+\sum_{n=2}^{\infty} \frac{1}{n+2} c_{n+1} \xi^{n+2} .
$$

Substituting (65) into (39), we obtain the exact analytic solution to (2) as follows:

$$
\begin{aligned}
u(x, t)=q \exp [ & p\left(\log x-\alpha v t^{2}\right) \\
& +\frac{1}{2} c_{1}\left(\log x-\alpha v t^{2}\right)^{2} \\
& +\frac{1}{3} c_{2}\left(\log x-\alpha v t^{2}\right)^{3} \\
& +\sum_{n=2}^{\infty} \frac{1}{n+2} c_{n+1}\left(\log x-\alpha v t^{2}\right)^{n+2} \\
& \left.+\frac{1}{2}(\alpha-\beta) v t^{2}-\frac{2}{3} \alpha v^{2} t^{3}+v t \log x\right],
\end{aligned}
$$

where $p=c_{0}$ and $q$ are arbitrary constants and the other coefficients $c_{n}(n=1,2, \ldots)$ are given by (62) and (63) successively.

Similarly, we can give the exact power series solution to (50) in the power series form (61). So, the exact analytic solution to (3) is obtained. The details are omitted here.

4.3. Exact Analytic Solution to (3). In Section 4.2, we construct the exact analytic solution to (49) by the power series method and obtain the exact analytic solution to (2). Now, we consider (53). Firstly, let $f^{\prime}=y$; then we get the following Riccati type of equation:

$$
\alpha \xi^{2} y^{\prime}+\alpha \xi^{2} y^{2}+\beta \xi y+\gamma \xi+v=0
$$

We will seek a solution of (67) in a generalized power series of the form

$$
y=A \xi^{-1}+\sum_{n=0}^{\infty} c_{n} \xi^{n}
$$

where the parameters $A$ and $c_{n}(n=0,1,2, \ldots)$ are constants to be determined. obtain

Substituting (68) into (67) and comparing coefficients, we

$$
A=\frac{(\alpha-\beta) \pm \sqrt{\Delta}}{2 \alpha}
$$

where $\Delta=(\alpha-\beta)^{2}-4 \alpha v$, and

$$
c_{0}=\frac{-\gamma}{2 \alpha A+\beta}, \quad 2 \alpha A+\beta \neq 0 .
$$

Generally, for $n \geq 0$, we have

$$
c_{n+1}=\frac{-\alpha}{(n+1) \alpha+2 \alpha A+\beta} \sum_{k=0}^{n} c_{k} c_{n-k}, \quad n=0,1,2, \ldots
$$


Thus, from (69) and (70), we can get $A$ and $c_{0}$. Furthermore, in view of (71), we have

$$
\begin{gathered}
c_{1}=\frac{-\alpha c_{0}^{2}}{\alpha+2 \alpha A+\beta}, \quad c_{2}=\frac{-2 \alpha c_{0} c_{1}}{2 \alpha+2 \alpha A+\beta}, \\
c_{3}=\frac{-\alpha\left(2 c_{0} c_{2}+c_{1}^{2}\right)}{3 \alpha+2 \alpha A+\beta},
\end{gathered}
$$

and so on (see Remark 3).

Therefore, the other terms of the sequence $\left\{c_{n}\right\}_{n=0}^{\infty}$ can be determined successively from (71) in a unique manner. This implies that for (67) there exists a generalized power series solution (68) with the coefficients given by (69)-(71). The convergence of the generalized power series solution (68) to (67) is similar to that in Section 4.2; we omit it in this paper. Thus, the power series solution (68) to (67) is also an exact analytic solution. follows:

Hence, the power series solution of (53) can be written as

$$
f(\xi)=\bar{c}+A \log |\xi|+c_{0} \xi+\frac{1}{2} c_{1} \xi^{2}+\sum_{n=1}^{\infty} \frac{1}{n+2} c_{n+1} \xi^{n+2}
$$

Substituting (73) into (52), we get the exact analytic solution to (3) as follows:

$$
\begin{aligned}
u(x, t)=c x^{A} \exp [ & c_{0} x+\frac{1}{2} c_{1} x^{2} \\
& \left.+\sum_{n=1}^{\infty} \frac{1}{n+2} c_{n+1} x^{n+2}+v t\right],
\end{aligned}
$$

where $c$ is an arbitrary constant and $A$ and $c_{n}(n=0,1,2, \ldots)$ are given by (69)-(71) successively.

Remark 1. We note that the generalized power series solution (68) differs from the regular form (61) since $A \neq 0$ in (68). In other words, there is no exact power series solution of the form (61) for (67). In particular, the determination of parameter $A$ depends on the equation greatly (cf. $[10,11]$ for details).

\section{Further Discussion about the General Bond Pricing Type of (1)}

In the above sections, we considered the symmetries, symmetry reductions, and exact solutions to the general bond pricing type of equation for the cases $\nu=0$ and $\nu=1$, which are the common forms in many practical applications, such as in financial mathematics. In this section, we discuss the generalized bond pricing type of equation of the form

$$
u_{t}+\alpha x^{2} u_{x x}+\beta x u_{x}+\gamma x^{\nu} u=0
$$

where $\nu \neq 0,1$ is an arbitrary positive number. Firstly, by the group classification method, we get the geometric vector field of (75) as follows:

$$
V_{1}=\partial_{t}, \quad V_{2}=u \partial_{u}, \quad V_{s}=s \partial_{u}
$$

where the function $s=s(x, t)$ satisfies (75).

Moreover, through the similarity transformation (42), we can reduce this equation to the following equation (ODE):

$$
\alpha \xi^{2} f^{\prime \prime}+\alpha \xi^{2} f^{\prime 2}+\beta \xi f^{\prime}+\gamma \xi^{\nu}+v=0
$$

where $f^{\prime}=d f / d \xi$. Similarly, we can consider the symmetry reductions and exact solutions to the equation. Now, as an example, we study the special case $v=2$. In this case, we have

$$
u_{t}+\alpha x^{2} u_{x x}+\beta x u_{x}+\gamma x^{2} u=0
$$

Referring to (77) and setting $f^{\prime}=y$, then we get the following reduced ODE of (78):

$$
\alpha \xi^{2} y^{\prime}+\alpha \xi^{2} y^{2}+\beta \xi y+\gamma \xi^{2}+v=0
$$

Suppose that (79) has the power series solution of the generalized form (68). Then, substituting (68) into (79) and comparing coefficients, we obtain

$$
A=\frac{(\alpha-\beta) \pm \sqrt{\Delta}}{2 \alpha}
$$

where $\Delta=(\alpha-\beta)^{2}-4 \alpha v$,

$$
\begin{aligned}
& (2 \alpha A+\beta) c_{0}=0, \\
& c_{1}=\frac{-\alpha c_{0}^{2}-\gamma}{\alpha+2 \alpha A+\beta} .
\end{aligned}
$$

Generally, for $n \geq 1$, we have

$$
c_{n+1}=\frac{-\alpha}{(n+1) \alpha+2 \alpha A+\beta} \sum_{k=0}^{n} c_{k} c_{n-k}, \quad n=1,2, \ldots
$$

In view of (81), we have two special cases as follows.

When $2 \alpha A+\beta \neq 0$, from (81), we have $c_{0}=0$. Furthermore, from (82) and (83), we have

$$
\begin{array}{ll}
c_{1}=\frac{-\gamma}{\alpha+2 \alpha A+\beta}, & c_{2}=0, \\
c_{3}=\frac{-\alpha c_{1}^{2}}{3 \alpha+2 \alpha A+\beta}, & c_{4}=0,
\end{array}
$$

and so on. In this case, by induction method, we have

$$
c_{2 n}=0, \quad n=0,1,2, \ldots
$$


When $2 \alpha A+\beta=0$, from (81), we get that $c_{0}$ is an arbitrary constant. Furthermore, from (82) and (83), we have

$$
\begin{array}{ll}
c_{1}=\frac{-\alpha c_{0}^{2}-\gamma}{\alpha+2 \alpha A+\beta}, & c_{2}=\frac{-2 \alpha c_{0} c_{1}}{2 \alpha+2 \alpha A+\beta}, \\
c_{3}=\frac{-\alpha\left(2 c_{0} c_{2}+c_{1}^{2}\right)}{3 \alpha+2 \alpha A+\beta}, & c_{4}=\frac{-2 \alpha\left(c_{0} c_{3}+c_{1} c_{2}\right)}{4 \alpha+2 \alpha A+\beta},
\end{array}
$$

and so on (see Remark 3).

Thus, the exact power series solutions to (79) are obtained. In view of (42), the exact analytic solutions to (78) are provided in power series form, respectively. More generally, for $\nu$ is an arbitrary positive integer, the exact power series solutions to (75) can be considered similarly by the generalized power series method; the details are omitted here.

\section{Conclusion and Remarks}

In this paper, we investigate the symmetry classifications and exact solutions to the bond pricing types of equations by the combination of Lie symmetry analysis and the generalized power series method; all of the exponentiated solutions and similarity solutions are obtained explicitly for the first time in the literature. Furthermore, for the generalized bond pricing type of equation, the vector field and exact solutions are provided simultaneously. These similarity solutions possess significant features in both financial problems and physical applications. On the other hand, it is known that tackling exact solutions to the vc-PDEs is a difficult problem; from the above discussion, we can see that the combination of Lie symmetry analysis and generalized power series method is a feasible approach and is worthy of further study.

Remark 2. Since there is no space translation $(x, t, u) \rightarrow$ $(x+\epsilon, t, u)$, the bond pricing equations have no traveling wave solutions. However, based on the exponentiated solutions, we can consider the other types of solutions, such as the fundamental solutions and sometimes iterative solutions [3$5,10]$.

Remark 3. In general, we cannot get the exact explicit solutions to the nonlinear equations such as (49), (53), and (79) by the classical analysis method. To tackle these equations, the generalized power series method and special techniques are necessary sometimes. For getting the exact analytic solutions in Sections 4.3 and 5, the condition $(n+1) \alpha+2 \alpha A+\beta \neq 0$ is necessary for $n=0,1,2, \ldots$.

\section{Acknowledgments}

This work is supported by the National Natural Science Foundation of China under Grant no. 11171041, the NSAF under Grant no. 11076015, and the Doctorial Foundation of Liaocheng University.

\section{References}

[1] R. K. Gazizov and N. H. Ibragimov, "Lie symmetry analysis of differential equations in finance," Nonlinear Dynamics, vol. 17, no. 4, pp. 387-407, 1998.

[2] C. A. Pooe, F. M. Mahomed, and C. Wafo Soh, "Invariant solutions of the Black-Scholes equation," Mathematical \& Computational Applications, vol. 8, no. 1-3, pp. 63-70, 2003.

[3] M. Craddock and E. Platen, "Symmetry group methods for fundamental solutions," Journal of Differential Equations, vol. 207, no. 2, pp. 285-302, 2004.

[4] M. Craddock and K. A. Lennox, "Lie group symmetries as integral transforms of fundamental solutions," Journal of Differential Equations, vol. 232, no. 2, pp. 652-674, 2007.

[5] M. Craddock and K. A. Lennox, "Lie symmetry methods for multi-dimensional parabolic PDEs and diffusions," Journal of Differential Equations, vol. 252, no. 1, pp. 56-90, 2012.

[6] W. Sinkala, P. G. L. Leach, and J. G. O’Hara, "Invariance properties of a general bond-pricing equation," Journal of Differential Equations, vol. 244, no. 11, pp. 2820-2835, 2008.

[7] J. Si, S. Chen, and P. Gong, "The FEM solution of valuation modal of TF convertible bonds," Journal of Huazhong University of Science and Technology, vol. 32, pp. 101-103, 2004 (Chinese).

[8] H. Liu, J. Li, and L. Liu, "Lie group classifications and exact solutions for two variable-coefficient equations," Applied Mathematics and Computation, vol. 215, no. 8, pp. 2927-2935, 2009.

[9] H. Liu, J. Li, and L. Liu, "Painlevé analysis, Lie symmetries, and exact solutions for the time-dependent coefficients Gardner equations," Nonlinear Dynamics, vol. 59, no. 3, pp. 497-502, 2010.

[10] H. Liu, J. Li, and Q. Zhang, "Lie symmetry analysis and exact explicit solutions for general Burgers' equation," Journal of Computational and Applied Mathematics, vol. 228, no. 1, pp. 1-9, 2009.

[11] H. Liu, J. Li, L. Liu, and Y. Wei, "Group classifications, optimal systems and exact solutions to the generalized Thomas equations," Journal of Mathematical Analysis and Applications, vol. 383, no. 2, pp. 400-408, 2011.

[12] H. Liu, J. Li, and L. Liu, "Complete group classification and exact solutions to the generalized short pulse equation," Studies in Applied Mathematics, vol. 129, no. 1, pp. 103-116, 2012.

[13] H. Liu and Y. Geng, "Symmetry reductions and exact solutions to the systems of carbon nanotubes conveying fluid," Journal of Differential Equations, vol. 254, no. 5, pp. 2289-2303, 2013.

[14] P. J. Olver, Applications of Lie Groups to Differential Equations, vol. 107 of Graduate Texts in Mathematics, Springer, New York, NY, USA, 1993.

[15] G. W. Bluman and S. C. Anco, Symmetry and Integration Methods for Differential Equations, vol. 154 of Applied Mathematical Sciences, Springer, New York, NY, USA, 2002.

[16] E. Pucci and G. Saccomandi, "Potential symmetries and solutions by reduction of partial differential equations," Journal of Physics, vol. 26, no. 3, pp. 681-690, 1993.

[17] C. Qu and Q. Huang, "Symmetry reductions and exact solutions of the affine heat equation," Journal of Mathematical Analysis and Applications, vol. 346, no. 2, pp. 521-530, 2008.

[18] T. Chaolu and P. Jing, "An algorithm for the complete symmetry classification of differential equations based on Wu's method," Journal of Engineering Mathematics, vol. 66, no. 1-3, pp. 181-199, 2010. 
[19] H. Liu and W. Li, "The exact analytic solutions of a nonlinear differential iterative equation," Nonlinear Analysis: Theory, Methods \& Applications, vol. 69, no. 8, pp. 2466-2478, 2008.

[20] N. Asmar, Partial Differential Equations with Fourier Series and Boundary Value Problems, China Machine Press, Beijing, China, 2nd edition, 2005. 


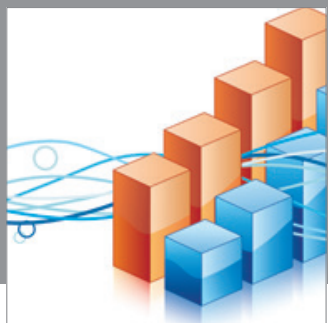

Advances in

Operations Research

mansans

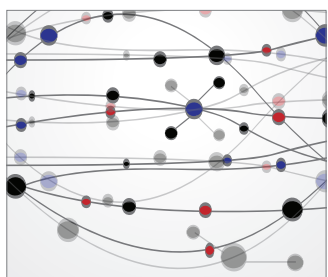

The Scientific World Journal
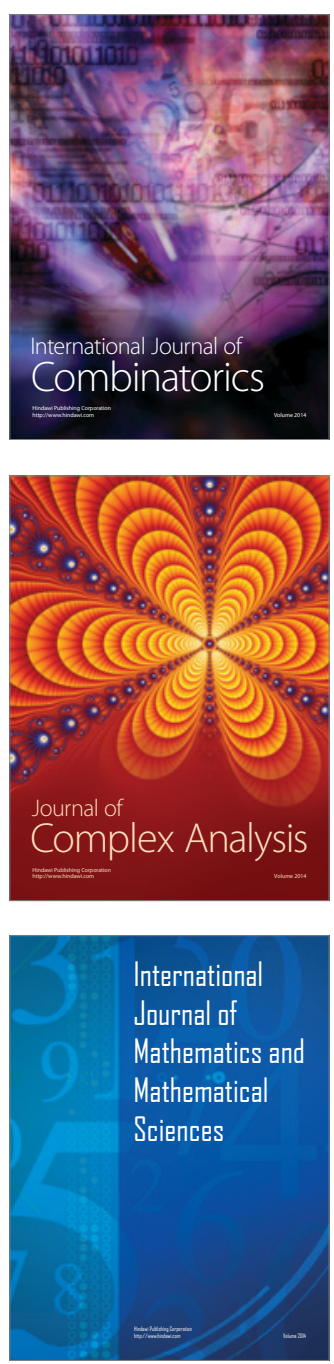
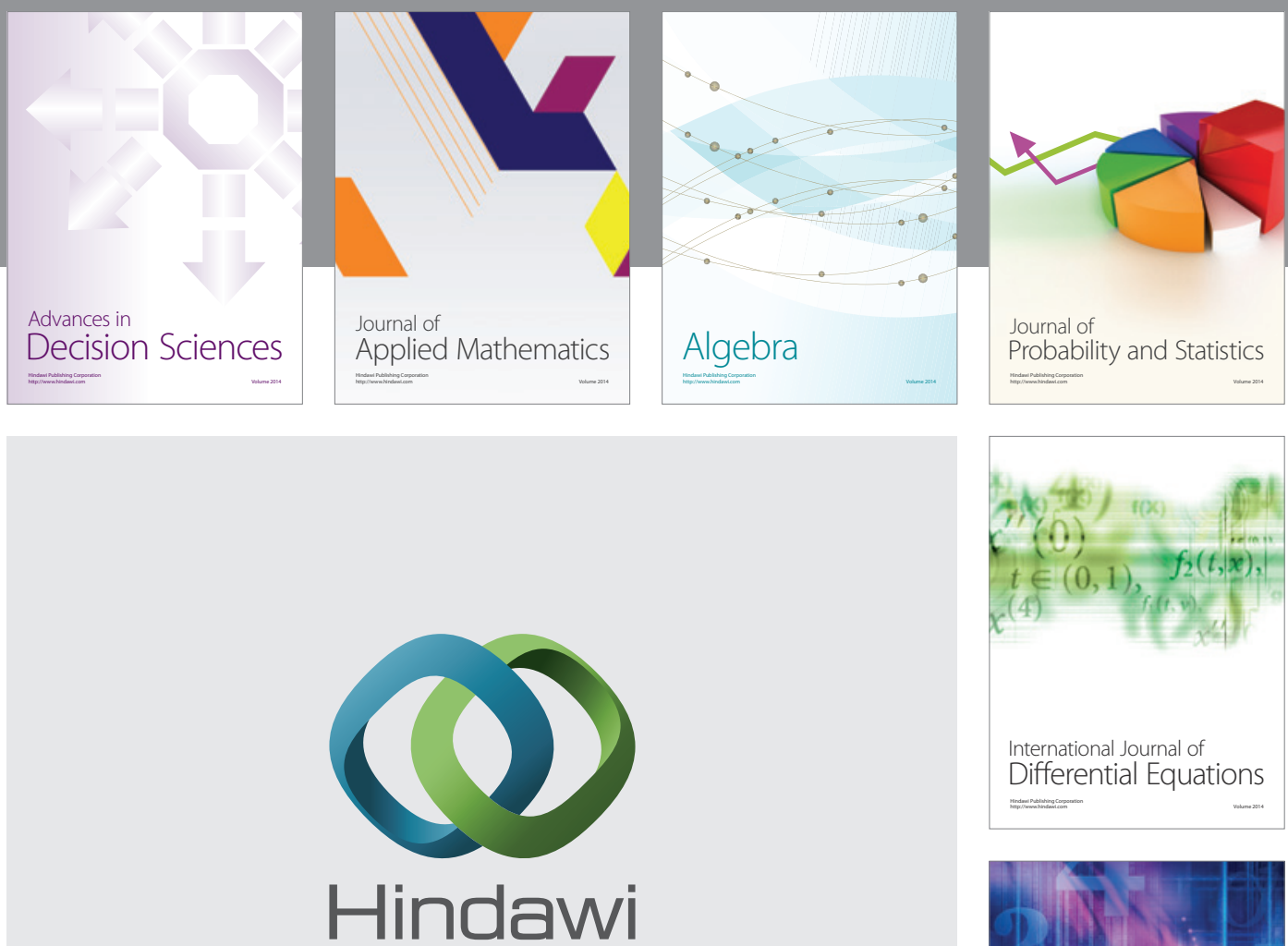

Submit your manuscripts at http://www.hindawi.com
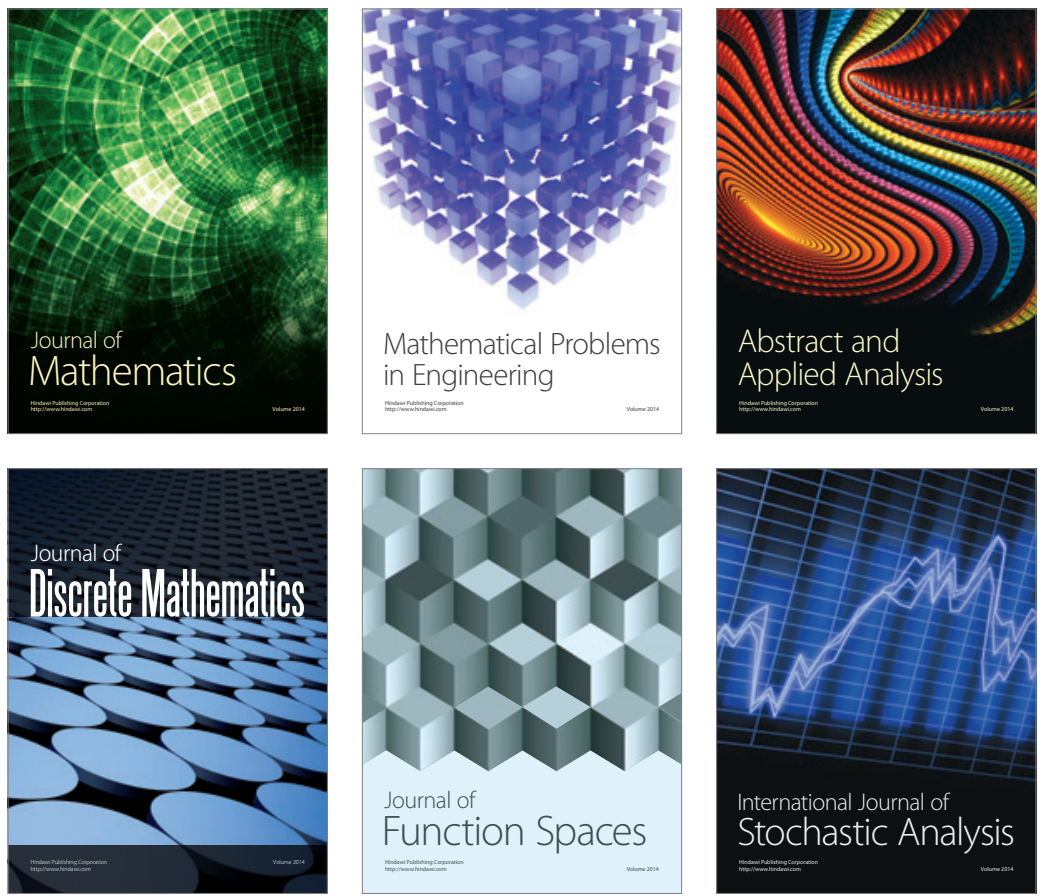

Journal of

Function Spaces

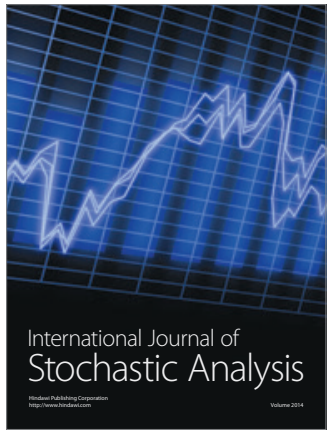

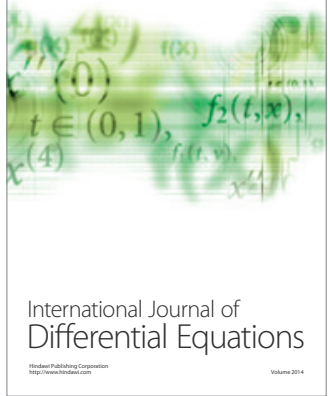
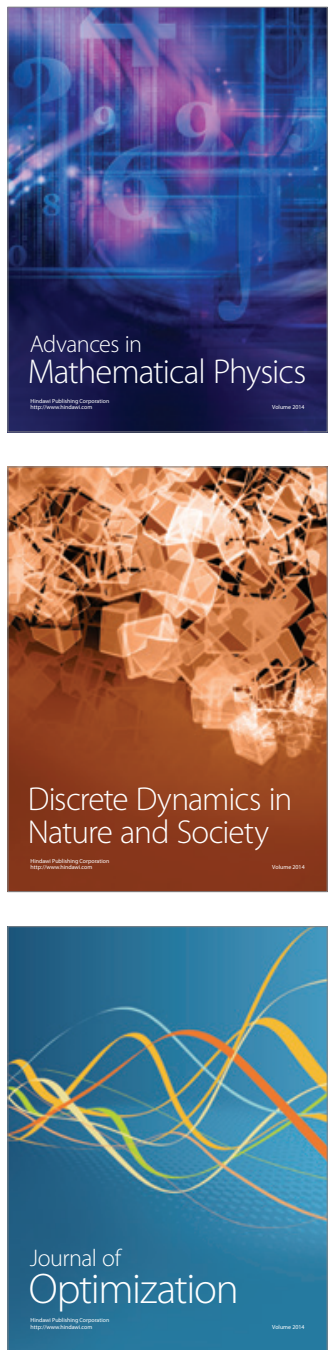